\title{
The Research and Field Application of Oil Displacement System for A Living Polymer
}

\author{
Guan Wang ${ }^{1, a}$,Ran Huo ${ }^{1, b}$,Jiliang $\mathrm{Yu}^{1, \mathrm{c}}$, Rui Wang ${ }^{1}$,Zhiqiang Wang ${ }^{1}$,Lisha \\ Duan $^{1}$,Shuai Wang ${ }^{2}$, Yajuan Yang ${ }^{3}$ \\ ${ }^{1}$ Engineering Technology Research Institute of Huabei Oilfield Company, Renqiu, Hebei, 062552 , \\ China \\ ${ }^{2}$ Down-hole operation of Bohai drilling Company, Renqiu, Hebei, 062552, China \\ ${ }^{3}$ The First Exploitation of Huabei Oilfield Company, Renqiu, Hebei, 062552, China \\ aemail:cyy_wangg@petrochina.com.cn, ${ }^{\text {bemail:cyy_huor@petrochina.com.cn, }{ }^{c} e m a i l: c y y \_y u l i l @ p e t r ~}$ \\ ochina.com.cn
}

Keywords: living polymer, interfacial tension, emulsion stability, oil displacement efficiency.

\begin{abstract}
: for the increasing cost of gel displacement and the influence of alkali corrosion and scaling of multivariate composite flooding on production equipment, a new type of living polymer was adopted. The living polymer has well heat resistance, salt tolerance and viscosifying capacity, and its molecular owns reactive group of special seepage characteristics. Both the characteristics of conventional polymer to improve the mobility ratio and the property of surfactant to reduce oil-water interfacial tension are possessed of this polymer. Through indoor physical and chemical performance evaluation and physical simulation experiment, the performance and oil displacement effect of the living polymer is studied. The results show that the living polymer has characteristics of better viscosifying capacity, salt resistance, emulsification and solubilization and so on. And then the field pilot testing was conducted of 6 water wells. The effective rate was $100 \%$ and the increased oil was 11069.1 tons, obtaining excellent effect.
\end{abstract}

\section{Introduction}

In Huabei oilfield, the main sandstone reservoir has stepped into high water cut late development stage, and gel displacement has become one of the main increase production measures ${ }^{[1-2]}$. But the high cost of gel displacement, the increasing rounds, and the single measures lead to worse measure effect year by year, which limit the further promotion of gel displacement. The conventional multivariate composite flooding technology achieve well application effect, but, at the same time, because of the effect of alkali corrosion and scaling on injection-production equipment and pipelines, the production and management cost are greatly increased ${ }^{[3]}$.

In order to meet the high continuous development requirements of complex fault block sandstone reservoir in Huabei oilfield, aiming at the existed problems, a living polymer of viscosifying capacity and surface activity is adopted, which has both the characteristics of polymer to improve the mobility ratio and the performance of the surfactant to reduce oil-water interfacial tension. By indoor test, the viscosifying capacity of living polymer and oil displacement efficiency are evaluated. Then the field pilot testing was conducted, which have guiding significance to improve the effect of sandstone reservoir development.

\section{The Molecular Structure Characteristics of Living Polymer}

The flexible hydrocarbon chain is the skeleton of living polymer with multivariate graft copolymerization, and the lipophilic group and hydrophilic group are joined on the molecular chain link. The polymer has a corresponding number of cationic functional groups and non-ionic structure unit. The chain internode and neighboring molecules have a strong interaction. Thus the living polymer has the dual properties of polymer and surfactant ${ }^{[4-5]}$. 


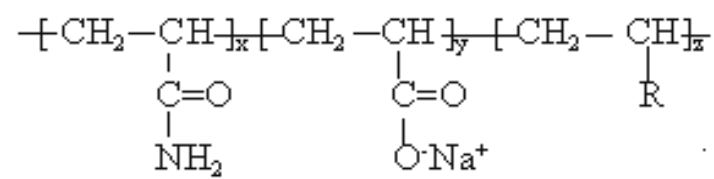

$\mathrm{R}=-\mathrm{OR},-\mathrm{NHR},-\mathrm{RSO}_{3} \mathrm{Na}$, - quaternary ammonium surfactant unit, - $(\mathrm{EO})_{\mathrm{n}}(\mathrm{PO})_{\mathrm{m}} \mathrm{R}$,

- cationic Gemini unit etal.

The living polymer molecular weight is not high, but the reactive group interaction is on the molecular link which produces strong effect on intermolecular, at the same time the hydration groups hydrate, leading to strong viscosifying capacity ${ }^{[6]}$.

\section{Indoor Performance Evaluations}

\subsection{Viscosifying capacity evaluation}

Experiment samples: living polymer, common polymer.

Experiment water: reinjection water of Baolige oilfield.

Experiment method: the two samples will be configured to different concentrations of aqueous solution, testing the viscosity under different concentrations. The experimental results are shown in figure 1 .

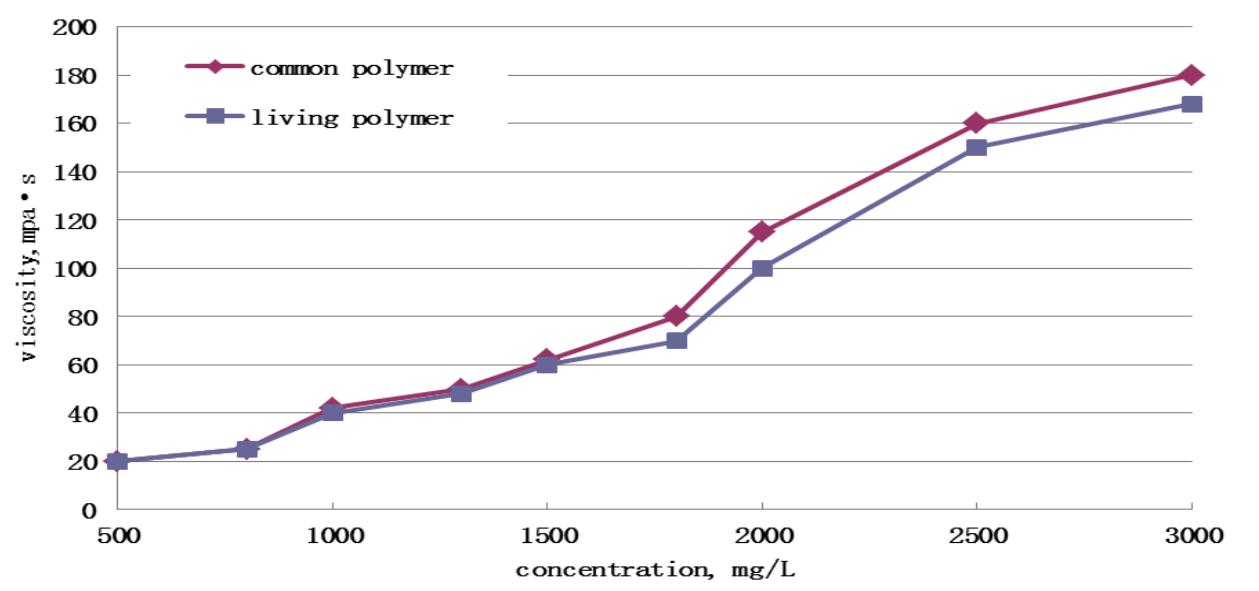

Figure. 1 the relation curve between viscosity and concentration

The experimental results show that the viscosity of living polymer aqueous solution increases over time at the beginning of aging, which is because the functional groups of poly-surfactant molecules gradually attract between others and cross-link, until the final balance. There exists hydrophobic associating in living polymer. Due to the presence of hydrophobic groups in aqueous solution, the hydrophobic association will generate, to minimize the chance to contact water, so that the system will stay on the lowest energy state. When in low concentrations, the living polymer aqueous solution are mainly composed of association within molecules, so the solution viscosity does not increase too much. With the increase of living polymer solution concentration, the intermolecular association is increased, and the living the polymer forms ordered self-assembly structure to a certain extent in solution, which is in the 'flake-mesh' multilayer stereoscopic mesh shape, leading to faster viscosity increasing.

\subsection{The solubilization performance evaluation}

Experiment samples: living polymer, common polymer, dehydrated crude oil from Bao 1 Combination Station.

Experiment water: reinjection water of Baolige oilfield.

Experiment method: The living polymer and polymer aqueous solution of $1000 \mathrm{mg} / \mathrm{L}$ are prepared respectively. $50 \mathrm{ml}$ of living polymer solution, polymer solution and reinjection sewage are poured into measuring cylinder respectively, then dehydrated crude oil is put into each 
measuring cylinder to $100 \mathrm{ml}$ and shake well. Placed under formation temperature of $55{ }^{\circ} \mathrm{C}$, observe the oil-water mixture state. The experimental results are shown in figure 2.

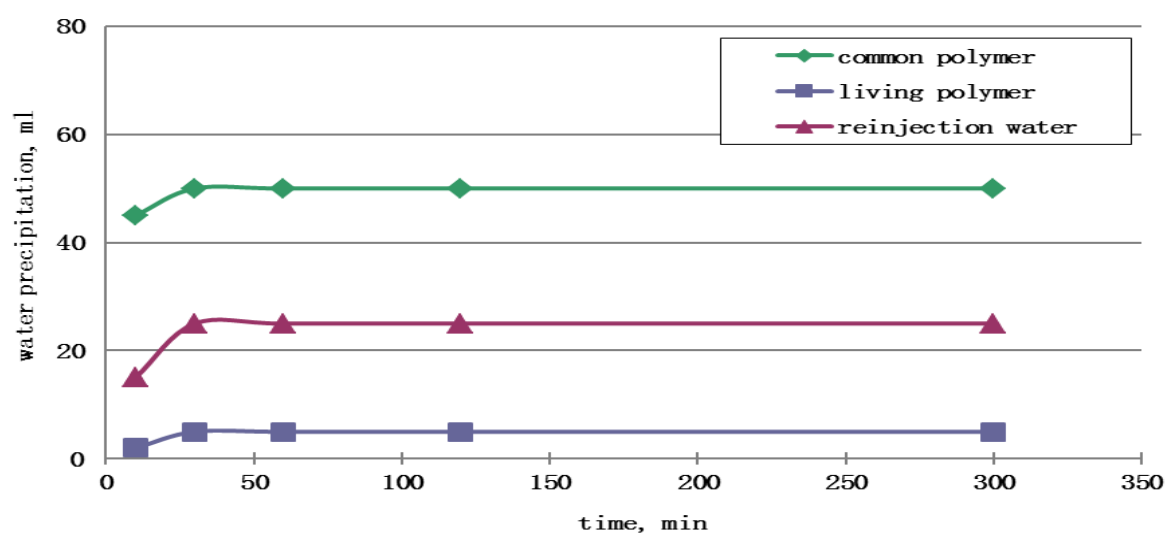

Figure. 2 the water precipitation of free water under $55^{\circ} \mathrm{C}$

The experimental results show that under the $55{ }^{\circ} \mathrm{C}$ formation temperature of the simulation of Baolige oilfield, water precipitation of different emulsions increase over time, and achieve stable after 3 hours. The increase range of emulsion water precipitation is the largest within 30 min when emulsion is forming. That is the best time for emulsion to coalescence. Water precipitation of living polymer emulsion is smaller than that of reinjection sewage emulsion and smaller than that of polymer emulsion. Therefore, living polymer has the property of compatibility with crude oil which polyacrylamide does not have. The emulsion stability is well, and after an hour the emulsion can achieve stability.

In $55^{\circ} \mathrm{C}$, under atmospheric pressure, the dispersed phase particle size of different emulsions was observed with a microscope, and the results are a shown in the figure below:

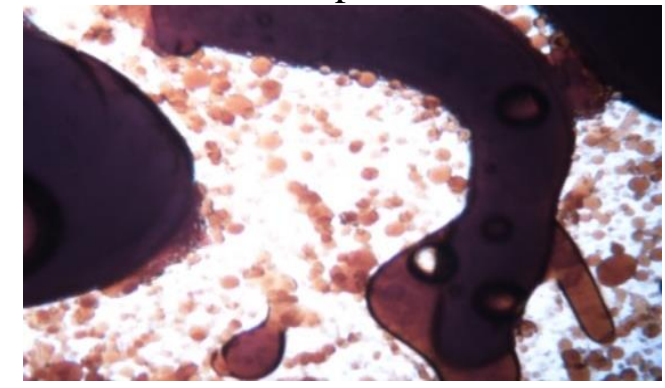

(a) polyacrylamide

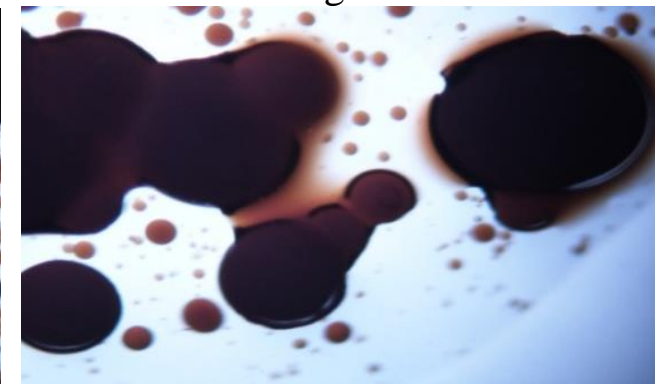

(b) living polymer

Figure. 3 The distribution map of the dispersed phase particle size for different emulsions

As shown in figure: the liquid shape of polyacrylamide emulsion system is highly irregular and distribute uneven. Figure (b) shows that the particle size of living polymer emulsion is small or big. The big one is coalescent oil droplet, the small one is oil droplet not be coalescent. After miscibility of living polymer and oil, the system has better stability.

\subsection{Interior displacement simulation experiment}

Experimental instruments and materials: physical model experiment equipment, constant temperature incubator $\left(55^{\circ} \mathrm{C}\right)$, quartz sand, output crude oil from Baolige oilfield, reinjection water from Baolige oilfield, etal.

Experimental conditions: core permeability is $100-300 \mathrm{mD}$, displacement under constant temperature of $55^{\circ} \mathrm{C}$, culture under constant temperature of $55^{\circ} \mathrm{C}$.

Experimental design: the size of core tube is $\Phi 2.5 \mathrm{~mm} \times 50 \mathrm{~cm}$. The core is vacuumzed and saturated with water, and then measure void volume and water phase permeability $\left(55^{\circ} \mathrm{C}\right)$. After being saturated with oil, the core is flooded by water until watercut is $98 \%$. Then inject $0.57 \mathrm{PV}$ polymers solution of $1200 \mathrm{mg} / \mathrm{L}$ and inject water subsequently until watercut is $98 \%$. After that, inject $0.57 \mathrm{PV}$ living polymers solution of $1200 \mathrm{mg} / \mathrm{L}$, and inject water subsequently until watercut 
is $98 \%$. Calculate the oil displacement efficiency and improved recovery factor. The results are shown in figure.

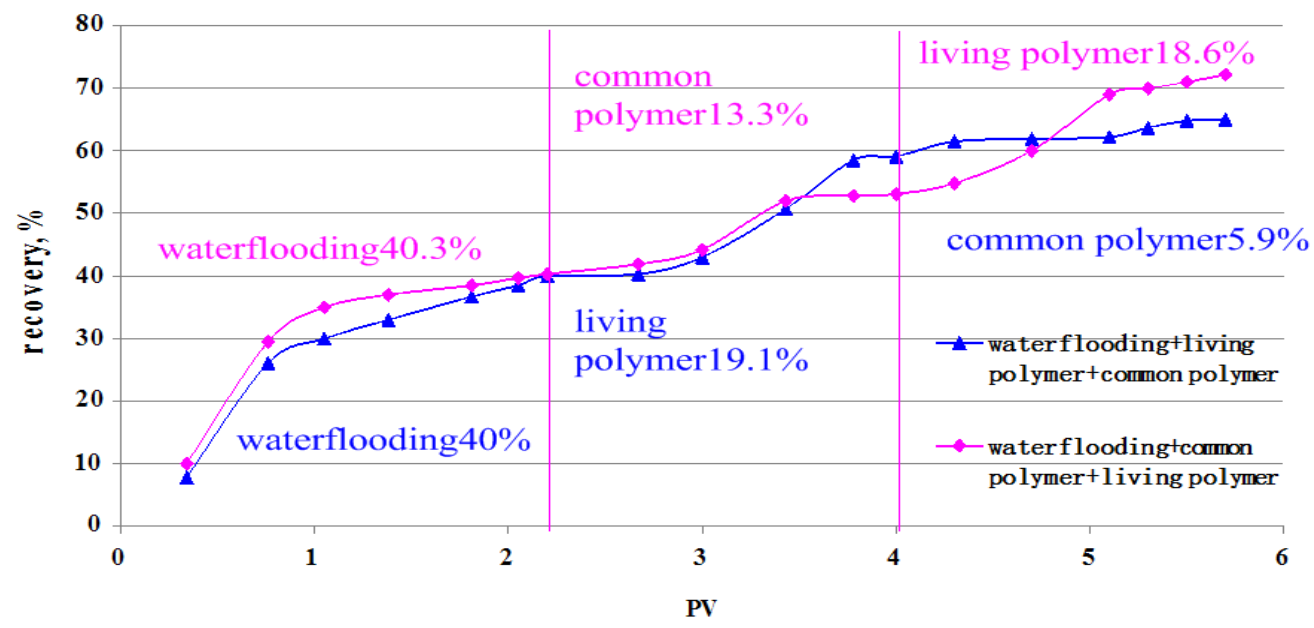

Figure. 4 The changing curves of recovery efficiency

Indoor physical model experiment results show that under the roughly same core parameters and basically the same primary waterflood recovery (about 40\%), the oil recovery of living polymer is superior to that of conventional polymer. After first waterflooding, the recovery can be improved by $19.1 \%$, while that of the common polymer is only $13.3 \%$. This excellent oil displacement effect is associated with lower surface activity of living polymer. At the same time, under the condition of same concentration and dosage, the recovery of injecting common polymer solution first and then living polymer solution is higher than that of injecting living polymer solution first and then common polymer solution.

\section{4. field implementation effect}

The field pilot testing was carried out in Ba 19 and 38 block of Baolige oilfield in September 2016. For the low permeability reservoirs and higher water injection pressure wells, first polymer and then living polymer injection method is used. For the better properties and higher permeability channel reservoirs, first gel and then living polymer injection pattern is adopted. 6 water wells were implemented on site. The total working liquid injection is $24,415 \mathrm{~m}^{3}$, and the success rate is $100 \%$. According to the comparison analysis of production data before and after measures, 21 oil wells corresponding to 6 water wells had oil increasing effect of different degrees. Till November 2017, watercut decreases from $88.6 \%$ to $80.9 \%$, the accumulative increased oil is 11069.1 tons, the average increased oil of single well 1844.8 tons, the increased oil rate is about $20 \%$. They are still in production increasing period.

\section{Conclusions}

(1) Living polymer has better properties of viscosifying capacity and solubilization. It also has certain surface activity except conventional polymer characteristics. So, the living polymer can achieve the effect of block, control and flooding at the same time in the formation.

(2) The indoor displacement simulation shows that the oil recovery can be increased by $18.6 \%$ if injecting living polymer after polymer flooding.

(3) The implementation results show that for different reservoir characteristics different living polymer injection ways can be chosen. By using this technology, sandstone reservoir development effect can be improved, achieving the result of decreasing water and increasing oil. 


\section{References}

[1] SONG She-min, WANG Ya-zhou, ZHOU Jun, eta. Early movable-gel-driving in water-flood development of heavy oil reservoirs [J]. Petroleum Exploration and Development, 2007, 34(5):585-589.

[2] LI Qiang, LU Xiang-Guo, XU Dian-Ping, eta. Study on Aggregate Structure of Polymeric Surfactant and its Influences on the Seepage Flow Characteristics [J]. Oilfield Chemistry, 2010, 27(4):398-402.

[3] YUAN Shi-yi, HAN Dong, MIAO Kun, eta. Application of flowing gel profile control technique to complex block reservoir [J]. Acta Petrolei Sinica, 2004, 25( 4):51-54.

[4] LU Xiang-guo, JIANG Wei-dong, WANG Xiao-yan. Study on effects of $\mathrm{Cr}^{3+}$, alkali and surfactant on polymer molecular configuration and seepage flow characteristics [J]. Acta Petrolei Sinica, 2009, 30(5):750-753.

[5] WANG Xian-jun, WANG Qing-guo. A Study on Inorganic Scale Formed and Scale Removing Fluid Used in Production Wells of ASP-Flooding Reservoirs [J]. Oilfield Chemistry, 2003, 20(1):1-3.

[6] WANG Xiao-yan, LU Xiang-guo, JIANG Wei-dong. Influences of cations, anions, anionic surfactants on the molecular coil dimension of partially hydrolyzed polyacrylamide [J]. Acta Polymerica Sinica, 2009, (12):1262-1265. 